\title{
La Curva en S como Herramienta para la Medición de los Ciclos deVida de Productos
}

\author{
Sergio Aguilar', Andrés F. Ávalos², Diana P. Giraldo³, Santiago Quintero ${ }^{4}$, Jhon W. Zartha ${ }^{5}$, \\ Farid B. Cortés ${ }^{6}$.
}

\begin{abstract}
The aim of this article was to carry out a study of the life cycles of three products of Colombian companies; based on a logistic model of population growth as a life cycles measurement tool. We found that the products life cycles have a similar behavior to the population growth, according to an $\mathrm{S}$ curve. The inflection points of the curves were obtained by a nonlinear regression. These points might be used as a tool for strategic decision making in products, in terms of identifying key instants for launching technological innovations, investments and execute marketing strategies.
\end{abstract}

Keywords: S-curve; Innovation; Life Cycles; marketing strategies; inflection points.

\section{Resumen}

En este artículo se realizó el estudio de los ciclos vida de tres productos de empresas colombianas basados en un modelo logístico de crecimiento demográfico como herramienta de medición de ciclos de vida. Se comprobó que los ciclos de vida de los productos tienen un comportamiento análogo al del crecimiento de la población, formando una curva en S. Adicionalmente se obtuvo por medio de una regresión no lineal los puntos de inflexión de las curvas. Estos podrán ser usados como herramienta para la toma de decisiones estratégicas en los productos, en cuanto a: determinar momentos claves en el lanzamiento de innovaciones tecnológicas, realizar inversiones y ejecutar estrategias de mercadotecnia.

Palabras clave: Curvas en s; innovación; ciclos de vida; estrategias de mercadotecnia; puntos de inflexión.

1, 2,3,4,5Grupo de Política y Gestión Tecnológica, Escuela de Ingenierías, Universidad Pontificia Bolivariana, Circular I N. 70-0 I.

Tel.: +57 (4) 4488388 ext. 9894. Medellín, Colombia. E-mail: sergioaguilarug@gmail.com, avalosp@gmail.com, dianap.giraldo@upb.edu.co, santiago.quintero@upb.edu.co,jhon.zartha@upb.edu.co

${ }^{6}$ Grupo de investigación en Yacimientos de Hidrocarburos, Facultad de Minas, Universidad Nacional de Colombia Sede Medellín. E-mail: fbcortes@unal.edu.co 


\section{Introducción}

La innovación tecnológica y en especial el cambio tecnológico se han convertido en un instrumento vital para aumentar la productividad, por lo que las empresas han ido destinando más inversiones en este fin. Estas inyecciones de capital deben ser realizadas en un momento adecuado, según la etapa en la que se encuentre el producto, para poder así optimizar sus beneficios y lograr un máximo provecho de ellas (Zartha, 2010; Pérez, 1992; Pérez, 200I; Tidd y Bessant 2009; White y Bruton, 2007).

Para las empresas es importante conocer las condiciones en que se encuentran sus productos para crear un plan estratégico de inversiones y mercadotecnia. Este plan se puede realizar mediante el conocimiento del ciclo de vida del producto (CVP), empleando un modelo logístico de crecimiento demográfico (Sood y Tellis, 2005).

El análisis del ciclo de vida de un producto parte de graficar un parámetro de desempeño acumulado en el tiempo, presentando un comportamiento tipo curva en S. Las curvas en $\mathrm{S}$ encontradas están compuestas por cuatros fases, que permiten determinar el momento adecuado para realizar inversiones y la ejecución de estrategias de mercadotecnia.

Con base en las curvas en $\mathrm{S}$ y el punto de inflexión obtenido de la misma, se puede conocer el momento adecuado para realizar una innovación radical o incremental en el producto, buscando la competitividad del mismo en el mercado y aumentar la productividad de la empresa. El punto de inflexión indica el momento en el cual el desempeño del producto disminuye e inicia la etapa de madurez (Zartha, 2010; Pérez, 1992; Pérez, 200I; Tidd y Bessant 2009; White y Bruton, 2007). Para determinarlo se requiere de un modelo que describa este tipo de comportamientos. Actualmente, el modelo más usado para describir el comportamiento de CVP es el propuesto por Velhulst (modelo logístico de crecimiento poblacional), tomado de Poveda (2007).

Algunos autores han trabajado en el análisis de CVP, entre ellos se puede destacar a Pérez (1992, 200I, 2004), Fernández (2005), Kucharavy (2007; 2008) y Shilling (2010), quienes han trabajado y usado una analogía de comportamiento de curvas en $\mathrm{S}$ de los productos. Sin embargo, este tipo de análisis de CVP en la Industria colombiana aún no ha sido utilizado.
El objetivo de este trabajo es el uso de las curvas en $\mathrm{S}$ como una herramienta para la medición de los CVP en algunas empresas colombianas del sector marroquinero, químico y textil. A partir del cual se obtienen los puntos inflexión que posibilitará la generación de estrategias y toma de decisiones en cuanto a inversión y mercadotecnia.

\section{Modelo Logístico}

El modelo logístico, y sus variantes, han sido empleados para la modelización de procesos biológicos y demográficos, con el fin de realizar pronósticos poblacionales; igualmente han sido aplicados con éxito en múltiples investigaciones sobre la difusión tecnológica (Poveda, 2007; López, 2005). Sin embargo, en el estado del arte aún no se tienen registros de aplicación de este modelo logístico al CVP en Colombia.

El análisis del crecimiento logístico parte del fenómeno del crecimiento de las poblaciones en el tiempo y resulta de realizar dos suposiciones fundamentales en esta dinámica (Poveda, 2007):

$\mathrm{p}=$ El crecimiento vegetativo más las inmigraciones, si las hay, es en todo momento proporcional al número de individuos presentes.

$\mathrm{K}=\mathrm{El}$ número máximo de pobladores que pueden vivir en un espacio limitado y/o bajo condiciones externas restrictivas.

Esto se puede expresar como:

$$
\frac{d P}{d t}=P f(P)
$$

La anterior ecuación diferencial es una suposición común en los modelos poblacionales $y$ es conocida como "hipótesis de dependencia de densidad” (Zill, 2006).

Debido que la población no puede superar una cantidad máxima $\mathrm{K}$ (el medio ambiente cumple una función restrictiva) se tiene:

$$
\begin{array}{r}
P=K / \\
f(K)=0
\end{array}
$$


o sea que bajo estas circunstancias la población no aumenta, además si:

$$
P=0
$$

$$
f(0)=\gamma
$$

siendo $\gamma$ un coeficiente constante que representa las características propias de la población (Zill, 2006). La relación entre $\mathrm{K}$ y $\gamma$ está representada por una línea recta con pendiente negativa. De esta forma se tiene:

$$
f(P)=a_{1} P+a_{2}
$$

usando las condiciones expresadas en las ecuaciones (2) y (3)

$$
f(P)=\gamma-\frac{\gamma P}{K}
$$

si

$$
\gamma=m K
$$

donde, $\mathrm{m}=$ constante de proporcionalidad. Reemplazando en la ecuación (I)

$$
\frac{d p}{d t}=m P(K-P)
$$

La siguiente es la solución de la ecuación diferencial (6)

$$
P(t)=a+\frac{K}{1+e^{-\gamma(t+D / m)}}
$$

donde,

$\mathrm{P}(\mathrm{t})=$ población en el tiempo

$+\mathrm{K}=$ Asíntota superior de la curva, límite máximo de pobladores.

$\gamma=$ Parámetro de crecimiento.

$-\mathrm{D} / \mathrm{m}=$ Punto de inflexión
Al graficar la ecuación (8) se obtiene la Figura I, la cual es la gráfica de la curva de crecimiento logístico de una población que comienza en $\mathrm{P}(0)$ y tiene un número máximo de habitantes $\mathrm{K}$. 


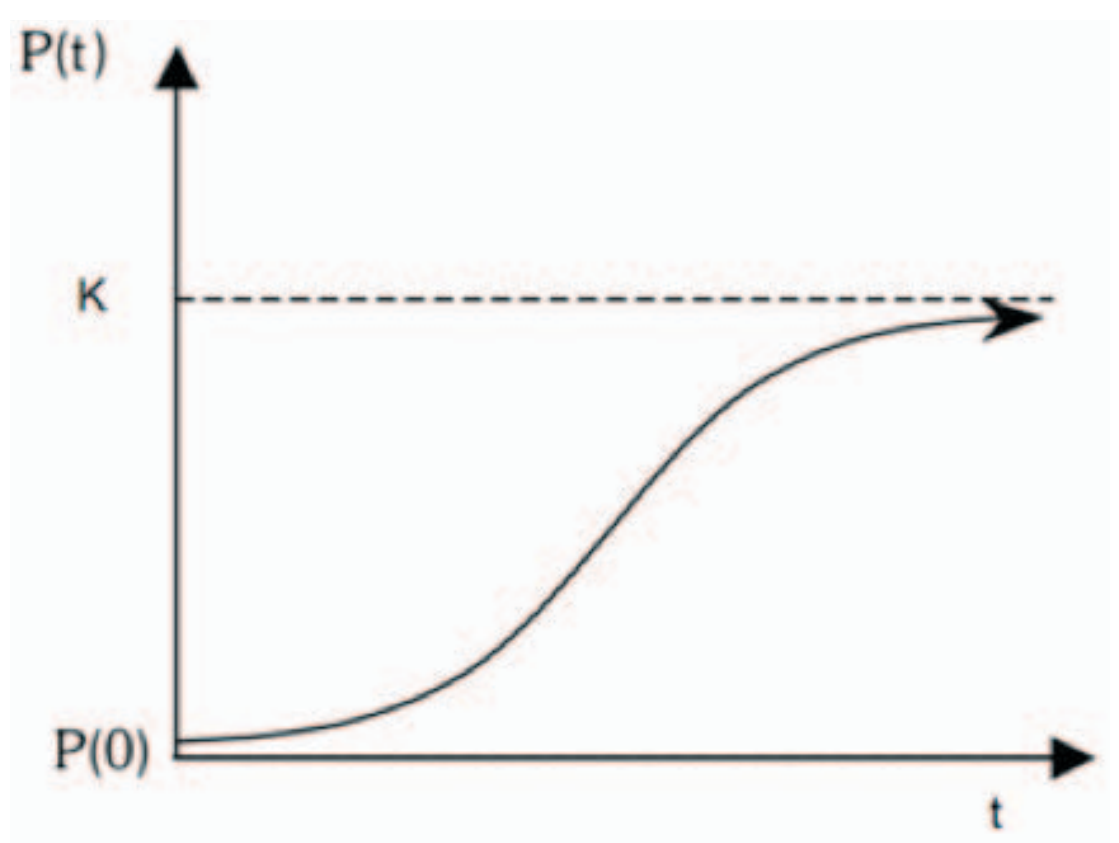

Figura I. Crecimiento de una población en el tiempo

\section{La Curva en S}

El CVP presenta un comportamiento análogo al reportado por el crecimiento poblacional. Con base en los parámetros de desempeño (eficiencia, ventas, número de usuarios, cantidad producida, entre otras) acumulados en el tiempo, se obtiene un comportamiento de curva en S. De esta forma se facilita el análisis de la difusión de la tecnología y se hace posible el estudio del CVP. Sin embargo, cabe destacar que para lograr mayor impacto en el proceso de toma de decisiones de las empresas innovadoras es importante tener en cuenta:

I) Analizar series de tiempos de mínimo 30 datos.

2) Analizar las curvas en $S$ de innovaciones tecnológicas de la misma línea.

3) En lo posible poder contar con datos de curvas en $\mathrm{S}$ del mismo sector al que pertenece la empresa.

4) Para las innovaciones tecnológicas analizadas es conveniente estudiar otro parámetro de desempeño (número de adoptantes/usuarios), con el fin de generar conclusiones sobre la eficiencia de cada uno.

\section{La curva en $\mathbf{S}$ y los ciclos de vida de los productos}

Con base en la analogía reportada entre el crecimiento poblacional y el CVP, este último presenta un comportamiento similar al de un ser vivo en todas sus etapas: nacimiento, crecimiento, reproducción y muerte “declive”. Así mismo, Pérez (1992) en sus análisis, señala cuatro partes fundamentales de la curva en "S", como se aprecia en la Figura 2: 


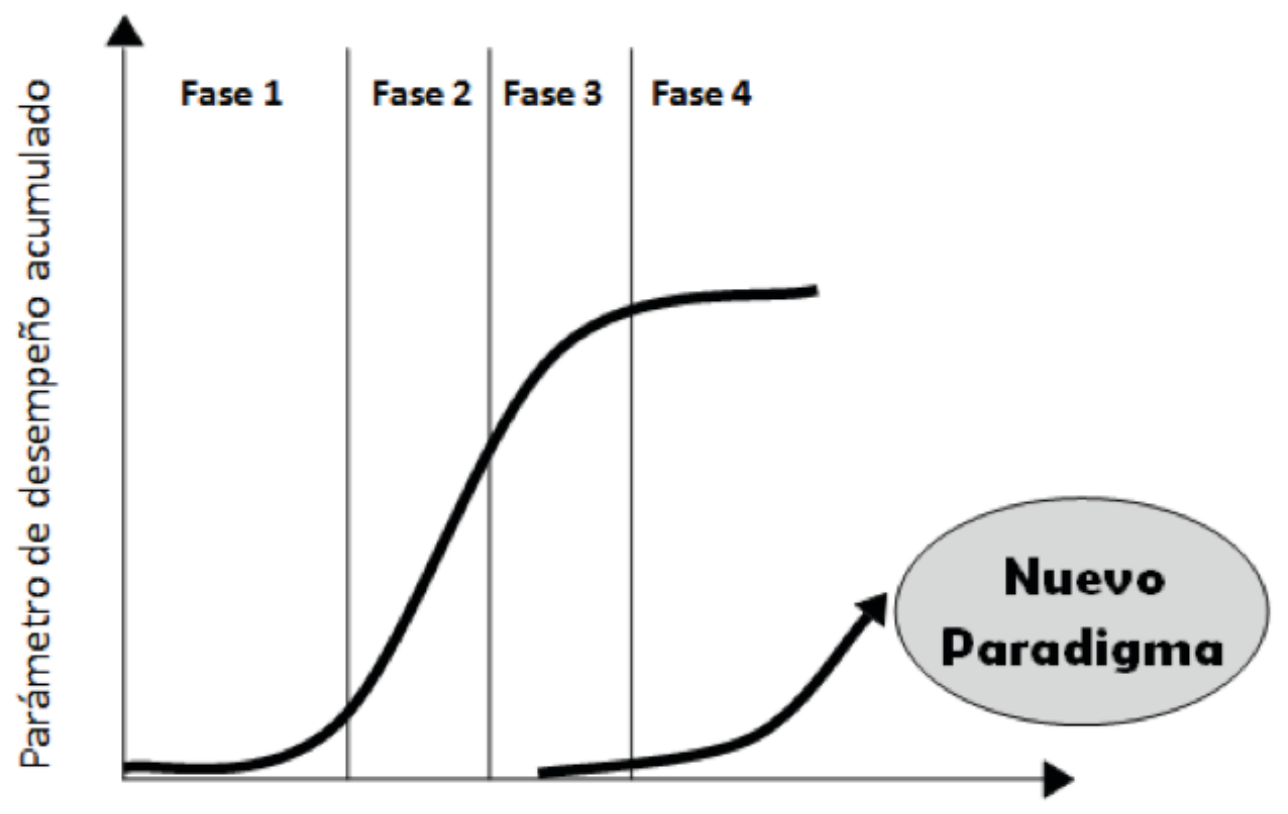

Figura 2. Partes de la curva en S (Pérez, 200I)

Fase I. Etapa de difusión inicial o de conocimientos públicos (Producto recién nacido): El producto en su etapa de lanzamiento presenta problemas de puesta a punto y a su vez participa en un mercado monopolístico u oligopolístico (Pérez, 1992, 200I). Cuando el producto se encuentra en esta fase es apreciado por su nivel de novedad y puede causar en los consumidores el entusiasmo suficiente como para que deseen tenerlo antes que los demás, aun sin conocer a fondo sus características (Shilling, 2010).

Fase 2. Etapa de rápido crecimiento (temprano): se caracteriza por la privatización del conocimiento, la experiencia técnica y el know-how (Pérez, 1992). En esta fase el producto se encuentra en pleno desarrollo: es en esta fase en donde se presenta una apertura en el mercado por medio de la aparición de nuevos competidores, se deben realizar inversiones masivas para adquirir o conservar una parte del mercado. El producto cuenta en esta etapa de un posicionamiento en el mercado. Todos los esfuerzos económicos de la empresa deben estar encaminadas a conservarla o aumentarla con el fin de no ceder terreno ante los competidores que cada vez son más numerosos, y la empresa encuentra ventajas competitivas en la capacidad de inversión en la expansión y en la fuerza de su red de distribución (Pérez, 1992).
Fase 3. Etapa de rápido crecimiento tardío: En esta fase aún se presenta la privatización del conocimiento, la experiencia técnica y el know-how (Pérez, 1992). El producto en esta etapa se encuentra en la madurez del ciclo de vida. A su vez se consolida la competencia y pocas empresas nuevas entran en el mercado. Los productos son muy rentables y exigen pocas inversiones para garantizar la presencia en el mismo. El crecimiento del mercado disminuye y las inversiones se limitan solo a las mejoras en los procesos. Se presenta una competencia fuerte para proteger la participación en el mercado. Además, la calidad del producto es un factor diferenciador (Pérez, 1992).

Fase 4. Etapa de conocimiento público: En esta fase se pasa de la saturación a la obsolescencia, lo que propicia la creación de un nuevo paradigma y por ende una nueva oportunidad tecnológica. El producto entra en etapa de envejecimiento y declive, y solo algunas empresas pueden conservar el producto en el mercado. Un punto clave en esta etapa, es el racionamiento de los gastos de producción haciendo énfasis en la relación costo eficiencia (Pérez, 1992). 


\section{La curva en $\mathbf{S}$ como herramienta de pronosticación}

El análisis de CVP es de suma importancia para las empresas, de modo que les permite estar preparados y recurrir a la pronosticación como herramienta para planificar estratégicamente las inversiones en innovación y mercadeo. Saffo (2007) se refiere a esto diciendo que "El arte de la pronosticación está en identificar una curva en $\mathrm{S}$ cuando comienza a emerger, mucho antes de su punto de inflexión”.

La tendencia en las innovaciones tecnológicas es a reducir el tiempo en los CVP (Ilegar a una obsolescencia temprana) debido a la exigencia del mercado. El punto clave para la toma decisiones y estrategias de mercadotecnia, debe partir de la información obtenida del análisis de los CVP, es decir, el punto de inflexión.

\section{Metodología}

Para este análisis se recurrió, inicialmente, a analogías que pudieran mostrar el camino más adecuado para tratar de medir la innovación tecnológica y el ciclo de vida de los productos, partiendo del análisis del crecimiento poblacional y del modelo que la describe. Posteriormente, se analizó la información preliminar, se trabajó en la consecución de los parámetros de desempeño, ventas en unidades de productos diferentes del sector industrial (Marroquinería, Químico y Textil), tal como se puede ver en la Tabla I.

\begin{tabular}{|l|l|l|}
\hline producto & sector & part etro de desempe- o \\
\hline Calzado & M arroquiner'a & Unidades vendidas \\
\hline Qu'mico & Qu'mico & Cantidad producida \\
\hline Ropa femenina & Textil & Unidades vendidas \\
\hline
\end{tabular}

Tabla I. Productos trabajados.

El modelo de crecimiento poblacional fue empleado debido a la similitud con el comportamiento descrito por el CVP. Además, se conocen el significado físico de cada uno de los parámetros que contiene el modelo de crecimiento poblacional aplicado al análisis de CVP (Sood y Tellis, 2005)

El modelo empleado en este artículo es:

$$
y(t)=a+\frac{b}{1+e^{-c(t-d)}}
$$

donde,

+b: límite del ciclo de vida del producto

c: Parámetro de crecimiento.

$\mathrm{d}$ : Punto donde las ventas en el tiempo comienzan a disminuir. (Punto de inflexión de la curva).

Para poder obtener los valores de las constantes fue necesario realizar una regresión no lineal.

\section{Resultados}

Se realizó una regresión no lineal para el conjunto de datos de ventas en el tiempo para cada uno de los sectores analizados, bajo los cuales y con la ayuda de un programa se pudo obtener cada uno de los valores de las constantes del modelo de crecimiento poblacional. Además de algunos parámetros estadísticos como el coeficiente de correlación, el error estándar y el valor estadístico Durbin-Watson.

A continuación se presentan los resultados obtenidos: Para el producto de marroquinería se obtuvo la curva en $\mathrm{S}$ mostrada en la Figura 3. La regresión no lineal de datos en el tiempo, arrojó como resultado el punto de inflexión en el día 20I.De lo cual se pude concluir que el tiempo adecuado para realizar una innovación debe ser inferior o igual a 201 días. 


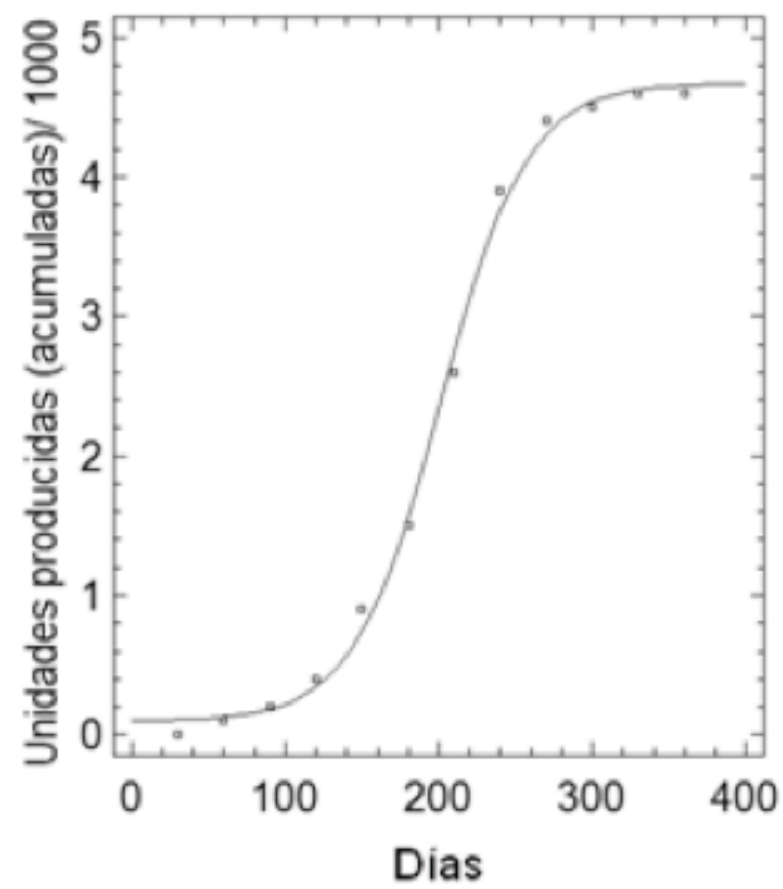

Figura 3. Ventas acumuladas en el tiempo del producto de marroquinería

En la Tabla 2 se presentan los resultados generales.

\begin{tabular}{|l|l|}
\hline & Producto marroquiner'a \\
\hline Punto de inflexin (d'as) & 201,023 \\
\hline a & 0,0973304 \\
\hline b & 4,57458 \\
\hline $\begin{array}{l}\text { Limite de vida del } \\
\text { producto }\end{array}$ & 4,6719104 \\
\hline Parłmetro de crecimiento & 0,0355026 \\
\hline R-cuadrado \% & 99,7629 \\
\hline Error estłndar & 0,113198 \\
\hline Durbin-Watson & 1,80055 \\
\hline Meses analizados & 12 \\
\hline
\end{tabular}

Tabla 2. Resultados del producto de marroquinería 
El estadístico $\mathrm{R}^{2}$ obtenido fue de $99,8 \%$, lo que indica un buen ajuste entre los datos experimentales y los teóricos. El estadístico Durbin-Watson (DW) examina los residuos para determinar si hay alguna correlación significativa, en este caso el DW = I,8 y el tamaño de la muestra es 12 . Teniendo en cuenta que el nivel de significancia es 0.05 se puede concluir que no existe autocorrelación serial entre los residuos.

El límite de vida del producto se presentó a las 467I unidades. En este punto, la demanda es baja y la obsolescencia puede causar el nacimiento de un nuevo paradigma, el cual surgirá a partir de la información técnica que se obtuvo del presente ciclo de vida.

La curva en $\mathrm{S}$ del producto químico se puede ver en la figura 4. El punto de inflexión ocurrió a los 492 días. De lo cual se pude concluir que el tiempo adecuado para realizar una innovación debe ser inferior o igual a 492 días. Los resultados obtenidos con este producto se presentan en la tabla 3.

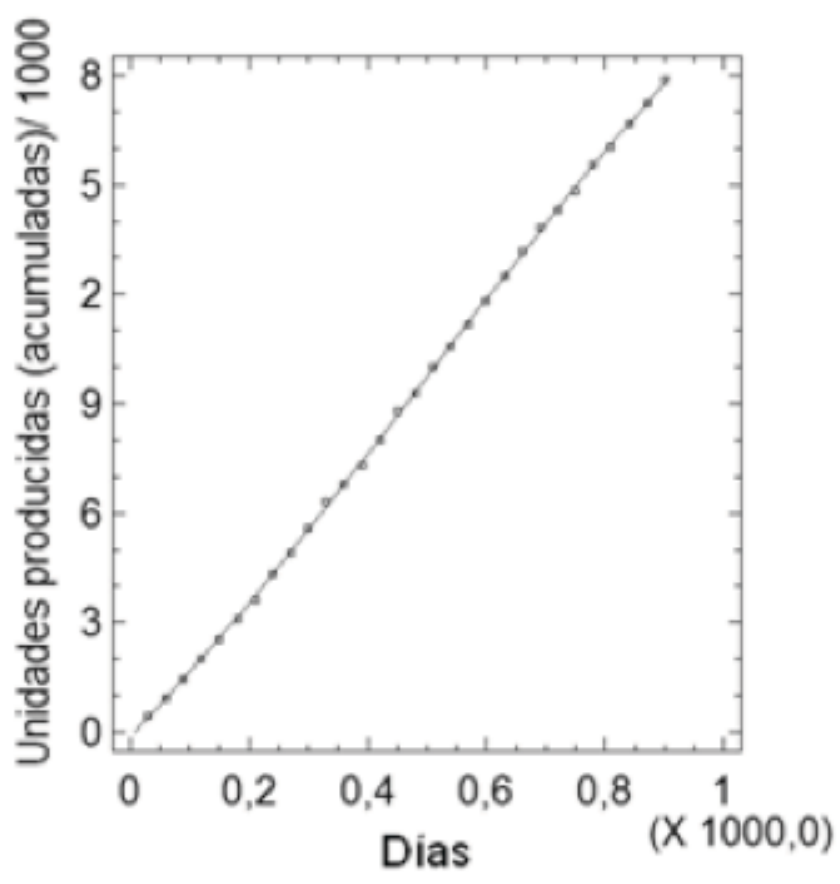

Figura 4. Producción acumulada en el tiempo del producto químico

\begin{tabular}{|l|l|}
\hline & producto qu'mico \\
\hline Punto de inflexi-n (d'as) & 492,145 \\
\hline a & $-11,6104$ \\
\hline b & 42,3629 \\
\hline $\begin{array}{l}\text { Limite de vida del } \\
\text { producto }\end{array}$ & 30,7525 \\
\hline Parłmetro de crecimiento & 0,00200884 \\
\hline R-cuadrado \% & 99,9863 \\
\hline Error estłndar & 0,066689 \\
\hline Durbin-Watson & 1,67637 \\
\hline M eses analizados & 30 \\
\hline
\end{tabular}

Tabla 3. Resultados del producto químico

El estadístico R2 obtenido fue de $99,98 \%$, lo que indica un buen ajuste entre los datos experimentales y los teóricos. El estadístico Durbin-Watson (DW) examina los residuos para determinar si hay alguna correlación significativa, en este caso el DW = 1,68 y el tamaño de la muestra es 30 . Teniendo en cuenta que el nivel de significancia es 0.05 se puede concluir que no existe autocorrelación serial entre los residuos.

El límite de vida del producto se presentó a las 30752 unidades. En este punto, la demanda es baja y la obsolescencia puede causar el nacimiento de un nuevo paradigma, el cual surgirá a partir de la información técnica que se obtuvo del presente ciclo de vida.

La curva en $\mathrm{S}$ para el producto textil se muestra en la Figura 5, el punto de inflexión ocurrió a los 139 días. De lo cual se pude concluir que el tiempo adecuado para realizar una innovación debe ser inferior o igual a 139 días. Los resultados de este producto se registraron en la tabla 4. 


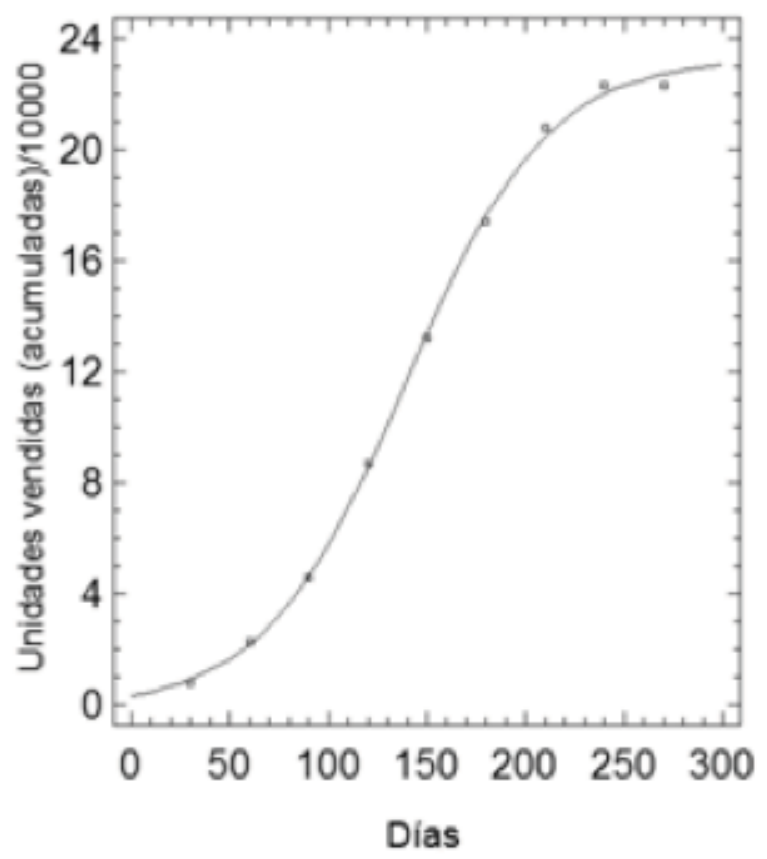

Figura 5. Ventas acumuladas en el tiempo del Producto textil

\begin{tabular}{|l|l|}
\hline & Producto textil \\
\hline Punto de inflexin (d'as) & 138,955 \\
\hline a & $-0,158226$ \\
\hline b & 23,461 \\
\hline $\begin{array}{l}\text { Limite de vida del } \\
\text { producto }\end{array}$ & 23,302774 \\
\hline Parłmetro de crecimiento & 0,0278579 \\
\hline R-cuadrado \% & 99,9132 \\
\hline Error estłndar & 0,323578 \\
\hline Durbin-W atson & 2,06337 \\
\hline M eses analizados & 270 \\
\hline
\end{tabular}

Tabla 4. Resultados del Producto textil 
El estadístico $\mathrm{R}^{2}$ obtenido fue de $99,98 \%$, lo que indica un buen ajuste entre los datos experimentales y los teóricos. El estadístico Durbin-Watson (DW) examina los residuos para determinar si hay alguna correlación significativa, en este caso el $D W=2,06$ y el tamaño de la muestra es 9 . Teniendo en cuenta que el nivel de significancia es 0.05 se puede concluir que no existe autocorrelación serial entre los residuos.

El límite de vida del producto se presentó a las 233027 unidades. En este punto, la demanda es baja y la obsolescencia puede causar el nacimiento de un nuevo paradigma, el cual surgirá a partir de la información técnica que se obtuvo del presente ciclo de vida.

\section{Conclusiones}

La utilización de analogías de crecimiento poblacional para comprender el ciclo de vida de los productos (desarrollos tecnológicos e innovación tecnológica) se constituye en un acercamiento útil al entendimiento de aspectos claves en la gestión de la innovación tecnológica.

El conocimiento de los puntos de inflexión es una herramienta útil para la toma de decisiones y estrategias de mercadotecnia, en cuanto a: al lanzamiento de nuevos productos, mejoras en los existentes, tipos de estrategia de mercadeo a utilizar y tiempos para realizar procedimientos de derecho tecnológico y propiedad intelectual.

El parámetro de desempeño comúnmente adoptado por la industria Colombiana son las ventas. Este parámetro no es el mejor debido a que se ve afectado por las variables económicas externas a la empresa como: la inflación, el IPC, variación en los precios, entre otros. Cabe destacar la importancia de que las empresas manejen otro tipo de parámetros de desempeño como: usuarios, horas hombre por producto, eficiencias, entre otras.

Los tiempos transcurridos entre innovaciones debe ser cada vez más cortos. Lo anterior tiene un límite, debido a que se puede llegar a un punto donde los ciclos sean demasiado cortos y el lanzamiento de innovaciones sea insostenible. En estos casos se puede mantener constante el tiempo entre innovaciones pero se debe buscar aumentar el desempeño de los productos, de tal forma que la pendiente en las fases I y 2 sea más alta que la del ciclo anterior.

\section{Agradecimientos}

Los autores agradecen a COLCIENCIAS y a la Universidad Pontificia Bolivariana por su apoyo logístico y financiero, en especial por financiar el programa de Formación de Doctorados.

\section{Referencias}

FERNÁNDEZ, E., 2005. Estrategias de Innovación. Madrid: Ed. Thomson.

KUCHARAVY, D., 2007. Application of S-shaped curve. HAL Articles en ligne, [en línea]. En: http://hal.archivesouvertes.fr/hal-00282758/en/ [Consulta 29 octubre 2009].

KUCHARAVY, D.y De Guio R., 2008. Logistic Substitution Model and Technological Forecasting. HAL Articles en ligne, [en línea]. En: http://hal.archives-ouvertes.fr/hal00338105/en/ [Consulta 29 octubre 2009].

LÓPEZ, J. y Arroyo J., 2005. Modelos matemáticos de difusión tecnológica. [En línea] Gijón: IX Congreso de Ingeniería de Organización. En: http://io.us.es/cio2005/ items/ponencias/30.pdf [Consulta 29 octubre 2009].

OECD., 2005. Manual de Oslo. Guía para la recogida e interpretación de datos de innovación. [En línea] OECD European Comunities. En: http://www.conacyt.gob. sv/Indicadores\%20Sector\%20Academcio/Manual_de Oslo\%2005.pdf [Consulta 29 octubre 2009].

PÉREZ, C., 1992. Cambio técnico, restructuración competitiva y reforma institucional en los países en desarrollo. El Trimestre Económico, n 223, . 23-64.

PÉREZ, C., 200I. Cambio tecnológico y oportunidades de desarrollo como blanco móvil. Revista de la CEPAL, [on line] Dic., 75, pp. II5-I36. En: http://www.eclac. org/publicaciones/SecretariaEjecutiva/0/LCG2/50PE/ carlotaperez.pdf [Consulta 29 octubre 2009].

Pérez, C., 2004.Technological revolutions, paradigm shifts and socio-institutional change. [En línea]. En: http:// www.carlotaperez.org/papers/basic-technologicalrevolutionsparadigm.htm [Consulta 29 octubre 2009]. 
POVEDA, G. y Manrique, J., 2007. Aplicación de la curva logística a los censos de la ciudad de Medellín. Ecos de Economía, [on line] Oct., 25, pp. 7-60. En: http://www.eafit. edu.co/ecos/ed25/25-I.pdf [Consulta 29 octubre 2009].

SAFFO, P., 2007. Seis reglas para pronosticar eficazmente. Harvard Business Review, Julio, 48 (7), pp. 100-110

SHILLING, M., 2010. Strategic management of technological innovation. New York: McGraw-Hill.

SOOD, A. y Tellis, G., 2005. Technological Evolution and Radical Innovation. Journal of Marketing [on line] Jul., 69 (3), pp. 152-168. En : http://www-rcf.usc.edu/ tellis/ radical.pdf [Consulta 29 octubre 2009].

TIDD, J., Bessant, J. 2009. MANAGING INNOVATION, Integrating Technological, Market and Organization Change, Jhon Wiley \& Sons Ltd., West Sussex,

WHITE, M; Bruton, G., 2007. The Management of Technology and Innovation: A Strategic Approach. Mason $\mathrm{OH}$ : Thomson Higher Education.

ZARTHA, J. W., Ávalos, A., AGUILAR, S., 2010. Curvas en $\mathrm{s}$, aplicación en productos innovadores del sector agroindustrial y químico colombiano.

ZILL, D. y Cullen, M., 2006. Ecuaciones diferenciales con problemas de valores en la frontera. Sexta ed. México D. F.: Thomson Learning. 
J. Technol. Manag. Innov. 20I2, Volume 7, Issue I 